\title{
SISTEMATIZAÇÃO DE UM PROTOCOLO DE ATENDIMENTO CLÍNICO ODONTOLÓGICO A GESTANTES EM UM MUNICÍPIO SUL CATARINENSE
}

\section{SYSTEMIZATION OF A PROTOCOL OF CLINICAL DENTAL CARE TO PREGNANT WOMEN IN A CITY SOUTH CATARINENSE}

\author{
Dionis Brognoli Cechinel* \\ Wanessa de Medeiros Boff** \\ Renan Antônio Ceretta"* \\ Priscyla Waleska Simões ${ }^{* * * *}$ \\ Luciane Bisognin Ceretta**** \\ Fernanda Guglielmi Faustini Sônego
}

\begin{abstract}
RESUMO
Introdução: A assistência odontológica para gestantes ainda sofre barreiras, pelos mitos e inverdades de que o tratamento odontológico venha causar algum dano à formação do bebê, uma vez que, nesse período, a cavidade bucal sofre alterações importantes, tornando o pré-natal odontológico um exame necessário. Métodos: Foi elaborado um protocolo de atendimento a gestantes e entregue aos profissionais; antes da entrega foi aplicado um primeiro questionário e, transcorridos 60 dias, um segundo questionário foi aplicado para saber a eficácia e uso do protocolo. Resultados: 11 cirurgiões-dentistas participaram da pesquisa; a média de idade foi de 35,81 anos $( \pm 4,89)$. Por meio do $1^{\circ}$ questionário $100 \%(n=11)$ se sentem seguros em atender as pacientes gestantes. Passados 60 dias o $2^{\circ}$ questionário foi aplicado para analisar a satisfação quanto à utilização do protocolo. 55,50\% $(n=5)$ concordam que o protocolo deixou-os mais seguros em realizar os procedimentos. Para 55,30\% ( $n=6)$ o protocolo proporcionou satisfação profissional. Conclusão: O conhecimento dos cirurgiões-dentistas sobre as alterações que ocorrem na gravidez é importante para se realizar um atendimento mais seguro, assim, nosso trabalho mostrou que o uso de um protocolo clínico no dia a dia proporcionou mais segurança nos atendimentos, como também satisfação profissional aos cirurgiões-dentistas que utilizam o protocolo.
\end{abstract}

DESCRITORES: Protocolos clínicos • Cuidado pré-natal • Gestantes • Saúde Bucal • Doença Periodontal.

\section{ABSTRACT}

Introduction: Dental care for pregnant women still suffers barriers, by the myths and untruths that the dental treatment will cause some damage the formation of the baby. During this period the oral cavity undergoes major changes, making the dental Prenatal a necessary exam. Methods: A protocol of care for pregnant women and delivered to the professionals was drawn up before the delivery, a first questionnaire was applied and 60 days have elapsed after a second questionnaire was applied to know the effectiveness and use of the protocol. Results: 11 dental surgeons in the survey, the average age was 35.81 years $( \pm 4.89)$. Through the 1 st survey $100 \%(n=11)$ feel safe to attend pregnant patients. After 60 days the 2 nd questionnaire was applied to analyze the satisfaction regarding to the use of protocol, $55.50 \%(n=5)$ agree that the protocol left them safer to perform the procedures, $55.30 \%(n=6)$ that the protocol provided job satisfaction. Conclusion: Knowledge of dental surgeons about the changes that occur in pregnancy is important to realize a safer service; our study has shown the use of a clinical protocol for day-to-day provided more security in attendance, as well as job satisfaction to the dental surgeons who use the protocol.

DESCRIPTORS: Clinical protocols • Prenatal care • Pregnant women • Oral health • Periodontal disease.

* Graduando em Odontologia - Universidade do Extremo Sul Catarinense - Email: dionibrog@hotmail.com

** Graduanda em Odontologia - Universidade do Extremo Sul Catarinense - Email: wanessa.boff@live.com

*** Doutor em Ciências da Saúde. Professor e coordenador do Curso de Odontologia - Universidade do Extremo Sul Catarinense. Email: rce@unesc.net.

**** Doutora em Ciências da Saúde - Universidade do Extremo Sul Catarinense. Professora do Curso de Odontologia e Professora Pesquisadora no Programa de Pós-Graduação em Saúde Coletiva - Universidade do Extremo Sul Catarinense. Email: pri@unesc.net.

***** Doutora em Ciências da Saúde - Universidade do Extremo Sul Catarinense. Professora do Curso de Odontologia e Professora Pesquisadora no Programa de Pós-Graduação em Saúde Coletiva - Universidade do Extremo Sul Catarinense. Email: luk@unesc.net.

******* Mestre em Odontopediatria. Professora do Curso de Odontologia - Universidade do Extremo Sul Catarinense. Email: fgfsonego@unesc.net 


\section{N T R O D U Ç Ã O}

Atualmente o atendimento a gestantes vem se destacando na Odontopediatria, porém a assistência odontológica para esse público ainda é repleta de barrei$\operatorname{ras}^{1,2}$. Muitos mitos, crenças e inverdades deixam as gestantes inseguras quanto ao atendimento odontológico, pois acreditam que o tratamento venha a prejudicar seu bebê ou a formação dele, fatos estes que contribuem negativamente para a saúde bucal da mãe e, consequentemente, para seu futuro bebê. Por outro lado, alguns cirurgiões-dentistas também compartilhem dessa ideia e acabam não se sentindo preparados para atendê-las, postergando o tratamento ${ }^{3-5}$.

Uma vez que a cavidade bucal sofre importantes modificações durante a gravidez é essencial o acompanhamento odontológico nessa fase. Sabe-se que as alterações hormonais, físicas e psicológicas podem influenciar e contribuir para o desenvolvimento de doenças bucais como cárie, gengivite e periodontite. Quando tais doenças estão associadas a outros fatores, algumas pesquisas relatam que podem induzir o parto prematuro ou o baixo peso ao nascer ${ }^{6,7}$.

Estudos recentes mostram que o atendimento odontológico deve ser realizado no período gestacional, e que o pré-natal odontológico torna-se importante e fundamental para introduzir bons hábitos desde cedo na vida da criança. Ao iniciar o pré-natal odontológico, o profissional deve orientar sobre o atendimento odontológico, examinar os tecidos bucais, buscando identificar riscos à saúde, como também instruir sobre hábitos alimentares, a ingestão de açúcar e instrução de higiene. Se houver necessidade de intervenção, esta deverá ser realizada ${ }^{3,7}$.

Percebendo a importância do tema, esse artigo apresenta a elaboração de um protocolo de atendimento clínico odontológico à gestante, que foi aplicado a cirurgiões-dentistas, de um município sul catarinense.

\section{MÉTODOS}

O presente estudo foi aprovado pelo Comitê de Ética e Pesquisa em Seres
Humanos do local onde a pesquisa foi realizada, sob o protocolo $\mathrm{n}^{\circ} 807.751$. Trata-se de uma pesquisa de abordagem quali-quantitativa, e de análise descritiva, realizada em um município sul catarinense que, segundo o Instituto Brasileiro de Geografia e Estatística (IBGE), possuía em 2015, uma população estimada de 50.000 habitantes.

Na primeira etapa do estudo foi realizada uma revisão de literatura, nas bases de dados como Scielo, PubMed, ScienceDirect, MedLine que embasaram a construção de um protocolo básico a ser utilizado no atendimento clínico odontológico voltado às gestantes, abordando como assunto as principais alterações que as gestantes enfrentam nesse período, sua posição na cadeira odontológica, qual o anestésico mais indicado e as medicações indicadas e contraindicadas.

Na segunda etapa o projeto foi apresentado, em uma reunião, aos 11 cirurgiões-dentistas que atuam na Atenção Básica do município, para que tivessem ciência da pesquisa e para que assinassem voluntariamente o Termo de Consentimento Livre e Esclarecido, conforme prevê a resolução no 466/12 do Conselho Nacional de Saúde, e aplicado um primeiro questionário contendo perguntas abertas e fechadas para avaliar o conhecimento deles acerca do atendimento clínico voltado à gestante.

Na sequência, por meio de uma aula expositiva, foram apresentados aos participantes os assuntos abordados no protocolo, e estipulado que eles teriam 60 dias para aplicá-lo durante os atendimentos clínicos.

Passados os 60 dias, foi utilizado um segundo questionário estruturado baseado na escala Likert, com o intuito de avaliar a satisfação dos profissionais quanto ao uso do protocolo e verificar se este correspondeu favoravelmente à prática clínica, ofertando um atendimento odontológico mais seguro e tranquilo para o profissional e para a gestante.

Após a coleta das informações, foi elaborado um banco de dados no software StatisticalPackage for the Social Sciences (SPSS) versão 22, também utilizado para a análise estatística. Foi utilizado o teste
CECHINEL DB

BOFF WM

CERETTA RA

SIMÕES PW

CERETTA LB

SONNEGO FGF

SISTEMATIZAÇÃO

DE UM PROTOCOLO

DE atendimento

CLÍNICO

ODONTOLÓgICO A

GESTANTES EM UM

MUNICÍPIO SUL

CATARINENSE

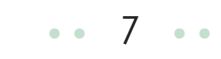

REV. ODONTOL.

UNIV, CID, SÃO

PAULO

2016; 28(1): 6-16, JAN - ABR 
CECHINEL DB

BOFF WM

CERETTA RA

SIMÕES PW

CERETTA LB

SONNEGO FGF

SI STEMATI ZAÇÃO

DE UM PROTOCOLO

DE ATENDIMENTO

CLÍNICO

ODONTOLÓGICO A

GESTANTES EM UM

MUNICÍPIO SUL

CATARINENSE

\section{SSN 1983-5183}

de Shapiro Wilk para avaliar a idade, tempo de trabalho e tempo de formação, que revelou distribuição gaussiana para essas variáveis; assim, foi calculada a média e o desvio-padrão para tais variáveis. Também foi estimada a frequência absoluta (n) e relativa (\%) para as variáveis qualitativas.

\section{RESULTADOS}

A amostra inicial foi composta por 11 cirurgiões-dentistas que trabalham nas Unidades Básicas de Saúde, no município quer trimestre e 9,10\% (n=1) não informaram; dados elucidados na Tabela 2.

Também foram questionados, quanto ao critério de escolha do uso de anestésico local, por meio de uma pergunta aberta: "Devemos ser seletivos quanto à escolha dos anestésicos locais a serem utilizados nas gestantes?". 37,00\% $(n=4)$ relataram que o melhor anestésico a ser utilizado é a lidocaína com epinefrina/adrenalina, dados apresentados na Figura 1. Quando questionados sobre a cautela na prescri-

Tabela 1. Características do perfil dos cirurgiões-dentistas que atuam na Atenção Básica de Saúde, do município, 2014.

\begin{tabular}{llr}
\hline \hline VARIÁVEL & $\mathrm{n}(\%)$ & \\
\hline \multirow{2}{*}{ Gênero } & $\mathrm{n}=(11)$ & $5(45,5)$ \\
& Feminino & $6(54,5)$ \\
Idade em anos & Masculino & 25 \\
& Mínima & 43 \\
& Máxima & $35,81( \pm 4,89)$ \\
& Média (Desvio-Padrão) & $12,36( \pm 5,23)$ \\
Tempo de serviço público - & Tempo de formado em anos - Média \\
Média (Desvio-Padrão) & (Desvio-Padrão) $( \pm 5,19)$ & $9(81,80)$ \\
Possui especialidade & Sim & $2(18,20)$ \\
\hline \hline
\end{tabular}

em estudo, sendo que a idade dos participantes variou entre 25 e 43 anos, com média de 35,81 $( \pm 4,89)$ anos.

Em relação ao tempo de formação, a média em anos foi de 12,36 ( $\pm 5,23)$, do tempo de serviço público foi de 9,60 $( \pm 5,19)$ anos, destes 11 participantes $81,80 \%(n=9)$ possuíam especialidades, dados estes expressos na Tabela 1. Por meio do $1^{\circ}$ questionário foi observado que $100 \%(n=11)$ dos cirurgiões-dentistas se sentem seguros em atender pacientes gestantes, porém verificou-se que um deles não realiza o atendimento. Ao mesmo tempo relatam a importância de um protocolo clínico para atendimento a gestante, assim como cursos de capacitações para atender esse público.

Cerca de $72,7 \%(n=8)$ relataram que o melhor período para realizar procedimentos odontológicos é no $2^{\circ}$ trimestre de gestação, 18,20\% (n=2) informou que o atendimento pode ser realizado em qual- ção medicamentosa, $100 \%(n=11)$ concordaram com essa questão.

Verificou-se que $100 \%(n=11)$ dos participantes afirmaram que a periodontite é um risco à gestação. Dos profissionais, $72,70 \%(n=8)$ acreditam que a periodontite ocorre com mais frequência na gestação, devido às alterações hormonais desse período, podendo ocasionar problemas descritos por estes na Figura 2. Dentre os entrevistados, $27,30 \%(n=3)$ descreveram que a periodontite independe da gestação e sim da higiene bucal.

Transcorridos os 60 dias, o $2^{\circ}$ questionário foi aplicado, buscando analisar a satisfação do atendimento clínico odontológico, propiciado pela utilização do protocolo e, dos 11 participantes da primeira etapa do estudo, apenas 9 profissionais responderam. Verificou-se que 1 deles estava de férias e o outro foi exonerado no período, dados estes expressos na tabela 3. 
Tabela 2. Análise do questionário 1 aos cirurgiões-dentistas que atuam na Atenção Básica de Saúde, do município, 2014.

\begin{tabular}{ll}
\hline \hline VARIÁVEL & $\mathrm{n}=(\%)$ \\
& $\mathrm{n}=11$
\end{tabular}

É realizado atendimento à gestante na Unidade Básica de Saúde

Sim

$10(90,90)$

Não

$1(9,10)$

Há grupos para Gestante na Unidade Básica de Saúde

Sim

$7(63,60)$

Não

$4(36,40)$

Sente-se seguro em atender gestantes

Sim

$11(100,00)$

Gestantes devem ser tradadas como pacientes especiais

Sim, pois apresentam maiores riscos no período.

$3(27,30)$

Não, devem ser tratadas normalmente.

$1(9,10)$

Depende do período.

Não respondeu.

$6(54,50)$

$1(9,10)$

Melhor período para atende-las

$2^{\circ}$ trimestre

$8(72,70)$

Todos os períodos

$2(18,20)$

Não respondeu.

$1(9,10)$

Há necessidade de posicionamento alternativo na cadeira odontológica.

Sim, durante toda a gestação

$1(9,10)$

Sim, durante $2^{\circ}$ e $3^{\circ}$ trimestres

$10(90,90)$

Os anestésicos devem ser selecionados

Sim

$9(81,80)$

Não

$2(18,20)$

Qual anestésico utilizar

Lidocaína/ Mepivacaína

$2(18,20)$

Lidocaína com epinefrina/ adrenalina

Mepivacaína com vasoconstritor

Mepivacaína sem vasoconstritor

Qualquer anestésico/evitar prilocaína

Não respondeu.

$4(36,40)$

$1(9,10)$

$1(9,10)$

$1(9,10)$

$2(18,20)$

Doença periodontal pode estar mais frequente na gestação.

Sim, devido a alterações nesse período

$8(72,70)$

Não, depende da higienização bucal da gestante

$3(27,30)$

Doença periodontal pode trazer riscos à gestação

Sim

$11(100,00)$

Justifique sua resposta

Parto prematuro e baixo peso ao nascer

$2(18,20)$

$5(45,50)$

$1(9,10)$

$1(9,10)$

Processos inflamatórios instalados

$2(18,20)$

Não respondeu.
CECHINEL DB

BOFF WM

CERETTA RA

SIMÕES PW

CERETTA LB

SONEGO FGF

SI STEMATIZAÇÃO

DE UM PROTOCOLO

DE ATENDIMENTO

CLÍNICO

ODONTOLÓGICO A

GESTANTES EM UM

MUNICÍPIO SUL

CATARINENSE
REV, ODONTOL.

UNIV. CID, SÃO

PAULO

$2016 ; 28(1): 6-16$ JAN - ABR 
CECHINEL DB
BOFF WM

SIMÕES PW :

CERETTA LB

SONNEGO FGF

SI STEMAT I ZAÇÃO DE UM PROTOCOLO

DE ATENDIMENTO

CLÍNICO

ODONTOLÓGICO A GESTANTES EM UM

MUNICÍPIO SUL

CATARINENSE
CERETTA RA

VARIÁVEL

$\mathrm{n}=(\%)$

$\mathrm{n}=11$

Cuidados na prescrição de medicamentos

Sim

$11(100,00)$

Justifique sua resposta

Problemas - pressão arterial

Algumas medicações

Medicação contraindicada

Interferência na formação da criança

Cautela na prescrição

Evitar antiinflamatórios

$1(9,10)$

$1(9,10)$

$2(18,20)$

$2(18,20)$

$1(9,10)$

$1(9,10)$

$1(9,10)$

$2(18,20)$

Medicamentos que rompem a barreira placentária

Não respondeu.

$3(27,30)$

$7(63,60)$

$1(9,10)$

Não, procedimento feito apenas no $1^{\circ}$ e $3^{\circ}$ trimestres

Perguntas frequentes feitas pelas gestantes aos cirurgiões-dentistas.

Cuidado com os filhos

$1(9,10)$

$1(9,10)$

Não recebe perguntas

$4(36,40)$

$3(27,30)$

Procedimentos que podem ser realizados/riscos para o feto.

$2(18,20)$

Curso de capacitação é importante

Sim

$11(100,00)$

Você acha relevante a criação de um protocolo de atendimento básico para o seu dia a dia.

Sim

$11(100,00)$

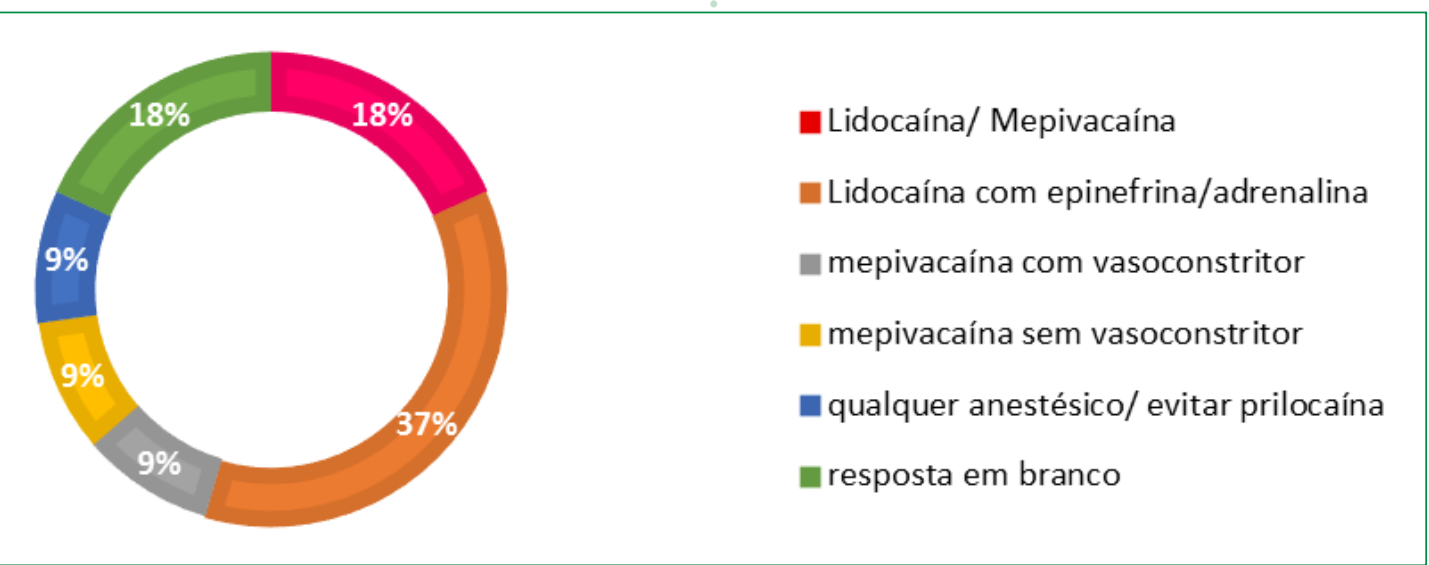

Figura 1. Percentual de cirurgiões-dentistas quainto à escolha do anestésico local a ser utiliza-

REV, ODONTOL.

UNIV, CID, SÃO

PAULO

2016; 28(1): 6-16, $J A N-A B R$

\section{do na gestante. \\ DI SCUSSÃO}

O objetivo principal do nosso estudo visou a sistematização de um protocolo clínico de atendimento básico à gestante, que proporcionasse satisfação aos profissionais e consequentemente mais segurança e qualidade no atendimento a esse público.

Participaram da pesquisa 11 cirurgi- 


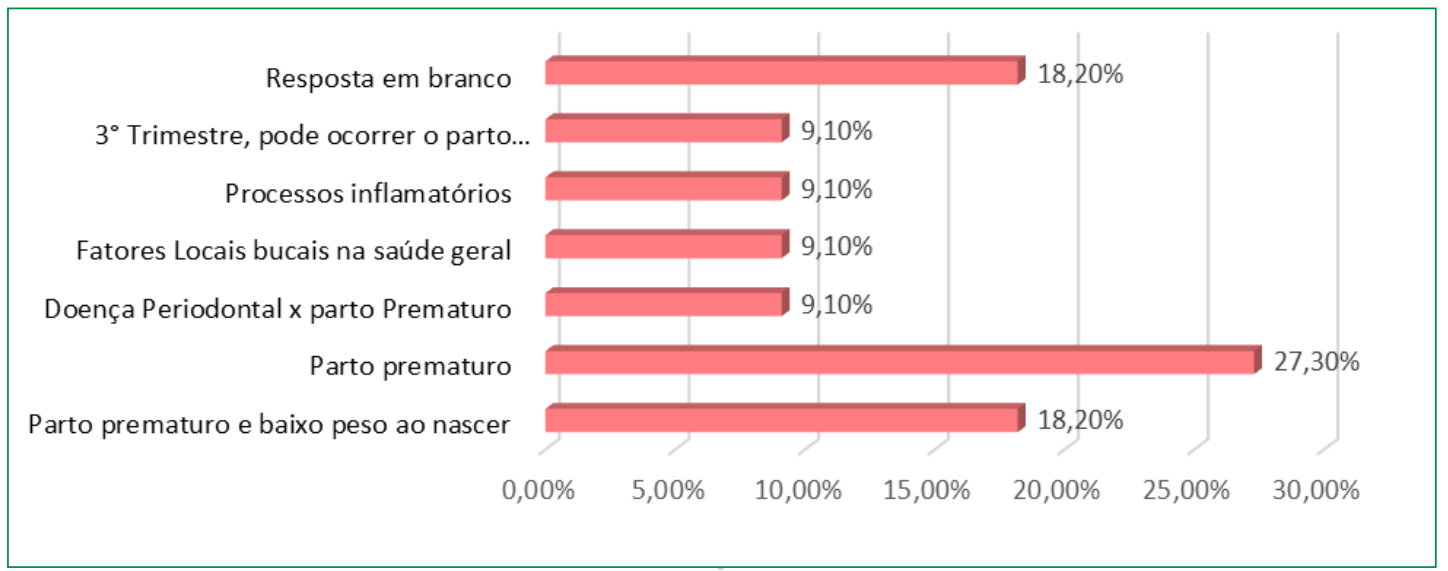

CECHINEL DB

BOFF WM

CERETTA RA

SIMÕES PW

CERETTA LB

SONEGO FGF

SI STEMATIZAÇÃO DE UM PROTOCOLO

DE ATENDIMENTO

CLINICO

ODONTOLÓGICO A

GESTANTES EM UM MUNICÍPIO SUL

CATARINENSE

Figura 2. Distribuição dos problemas relacionados à doença periodontal descritos pelos cirurgiões-dentistas.

Tabela 3. Análise de satisfação em utilizar o protocolo, dos cirurgiões-dentistas que atuam na Atenção Básica de Saúde do município, segundo o questionário 2.

\begin{tabular}{ll}
\hline \hline VARIAVEL & $\mathrm{n}=(\%)$ \\
& $\mathrm{n}=(9)$ \\
\hline
\end{tabular}

As informações contidas no protocolo foram de fácil entendimento.

Concorda inteiramente

$6(66,70)$

Concorda

As informações contidas no protocolo foram insuficientes.

Concorda

Concorda moderadamente

$1(1110)$

Neutro ou indeciso

Discorda

$5(55,60)$

A utilização do protocolo deixou-o mais seguro para realizar os procedimentos.

Concorda inteiramente

Concorda

Concorda moderadamente

Neutro ou indeciso

Discorda moderadamente

$1(11,10)$

Não tenho dúvidas: o que faço em meu trabalho é realmente importante.

Concorda inteiramente

Concorda

Neutro ou indeciso

Estou satisfeito com as atividades que realizo em meu trabalho.

Concorda inteiramente

Concorda

Neutro ou indeciso

$1(11,10)$

No meu trabalho, algumas vezes, tenho que fazer coisas que vão contra meu julgamento profissional.

Concorda moderadamente

Neutro ou indeciso

Discorda moderadamente $2(22,20)$

REV, ODONTOL.

UNIV, CID, SÃO

PAULO

2016; 28(1): 6-16, JAN - ABR 
CECHINEL DB BOFF WM

CERETTA RA

SIMÕES PW

CERETTA LB

SONNEGO FGF

SI STEMATIZAÇÃO DE UM PROTOCOLO

DE ATENDIMENTO

CLINICO

ODONTOLÓGICO A GESTANTES EM UM :

MUNICÍPIO SUL

CATARINENSE

REV. ODONTOL.

UNIV, CID, SÃO

PAULO

2016; 28(1): 6-16, JAN - ABR

VARIAVEL

$\mathrm{n}=(\%)$

Discorda

$3(33,30)$

Discorda inteiramente

$2(22,20)$

O protocolo não modificou em nada minha conduta profissional.

Concorda moderadamente

$1(11,10)$

Neutro ou indeciso

$2(22,20)$

$1(11,10)$

Discorda moderadamente

$2(22,20)$

Discorda

$3(33,30)$

Discorda inteiramente

As decisões administrativas da instituição interferem na assistência ao pacien-

te.

Concorda moderadamente

$1(11,10)$

Discorda moderadamente

Discorda

Discorda inteiramente

A utilização do protocolo superou minhas expectativas.

Concorda inteiramente

Concorda

Concorda moderadamente

$1(11,10)$

Neutro ou indeciso

$3(33,30)$

Discorda moderadamente

$1(11,10)$

Discorda

$1(11,10)$

A utilização do protocolo proporcionou satisfação profissional.

Concorda inteiramente

$1(11,10)$

Concorda

Concorda moderadamente

Neutro ou indeciso

Discorda

$1(11,10)$

Recomenda a construção de outros protocolos clínicos.

Concorda inteiramente

$3(33,30)$

Concorda

$4(44,40)$

Discorda

$1(11,10)$

Discorda inteiramente

$1(11,10)$

ões-dentistas, sendo que a idade dos profissionais variou entre 25 e 43 anos, com média de 38,81 $( \pm 4,89)$ anos. Quanto ao tempo de formação, a média foi de 12,36 $( \pm 5,23)$ anos e a do tempo no serviço público foi de 9,60 $( \pm 5,19)$ anos.

Em relação à segurança para atender as gestantes, foi observado em nossa pesquisa que $100 \%$ dos cirurgiões-dentistas apresentaram-se seguros em atender, porém observou-se que um deles não realiza atendimento, o que sugere falta de segurança ou desconhecimento. Nesse contexto, um estudo realizado pela Universidade Federal do Pará, em 2013, com 138 cirurgiões-dentistas, mostrou que a maioria dos profissionais entrevistados já realizou atendimento odontológico em gestantes e $12,30 \%$ demonstraram não ter conhecimento sobre o exame de pré-natal odontológico mas, mesmo assim, realizam o atendimento ${ }^{8}$. Corroborando com nosso trabalho, uma pesquisa realizada na Universidade de São Paulo, em 2008, com 37 profissionais, revelou que $73,00 \%$ dos dentistas sentiam-se seguros em rea- 
lizar o atendimento ${ }^{9}$. Outro estudo feito pela Faculdade de Odontologia de Verdun com 241 dentistas, em 2012, mostrou que $49,80 \%$ dos entrevistados recebem frequentemente mulheres grávidas em seus consultórios $^{10}$. Outros autores descrevem a importância do pré-natal odontológico e que este deve ser incluído como parte integrante da assistência à mulher grávida, fazendo parte da rotina na gravidez ${ }^{11}$.

A melhor época para atendimento à gestante foi questionada; assim, 72,70\% da nossa amostra relatou que o melhor período para atender as gestantes é o segundo trimestre, e 18,20\% informaram que o atendimento pode ser realizado em qualquer período. Outros estudos também concordam que o período mais seguro para atendimento a essas pacientes é o segundo trimestre da gestação ${ }^{8,11,12}$. Em contrapartida, há autores que falam que os procedimentos odontológicos como higienização bucal, extração, drenagem de abscessos e outros, podem ser realizados em qualquer período $9,10,13,14$.

Quanto ao uso de anestésico local, foi dirigida uma pergunta aberta: "Qual o critério de escolha para o uso de anestésico local em gestantes?". Dos participantes, $37,00 \%$ responderam que a lidocaína com epinefrina/adrenalina é a mais indicada. Corroborando com nossa pesquisa, um estudo realizado no Pará, em 2013, com os cirurgiões-dentistas, que foram questionados também com uma pergunta aberta, responderam predominantemente lidocaína $(45,00 \%)^{8}$. Nesse contexto, outro estudo realizado em São Paulo, em 2011, mostra que o anestésico de escolha por $42,20 \%$ dos dentistas foi lidocaína + epinefrina $1: 100.000^{15}$.

Vários estudos relatam os riscos da doença periodontal associada à gravidez, assim como em nossa casuística em que $100 \%$ dos entrevistados afirmaram que a periodontite é um risco à gestação, e $72,70 \%$ sugeriram que a periodontite é mais frequente na gestação, devido às alterações hormonais que ocorrem no período. Os respondentes sugeriram também que a periodontite possa influenciar o parto prematuro $(45,50 \%)$ ou parto prematuro e baixo peso ao nascer $(18,20 \%)$. Quando questionados se a doença perio- dontal pode estar mais frequentemente associada à gestação, 27,70\% responderam que não depende de estar grávida para isso e, sim, da própria higiene bucal. Tais fatos também se encontram no trabalho realizado pela Faculdade de Odontologia de Verdun (2012), em que 84,90\% dos dentistas acreditam que uma infecção oral pode representar algum risco na gestação, $53,60 \%$ descrevem o risco ao parto prematuro e $45,00 \%$ do parto prematuro e baixo peso ao nascer em bebês ${ }^{11}$.

Num estudo realizado no departamento de Odontologia em Saúde Coletiva na Índia em 2014 com 130 obstetras entrevistados, verificou-se que $80,00 \%$ dos participantes consideraram a relação entre a saúde gengival e a gravidez, 85,00\% afirmaram que a periodontite na gravidez pode causar um eventual parto prematuro e 75,00\% afirmaram que pode causar parto prematuro e baixo peso ao nascer ${ }^{11,12,16}$. Outro trabalho mostrou que $33,00 \%$ dos profissionais revelaram que a gengivite no período gestacional foi decorrente das alterações hormonais e 33,00\% a atribuíram à higiene deficiente ${ }^{8}$.

O uso de medicamentos na gravidez representa um desafio para os profissionais, pois pode implicar em danos tanto para mãe quanto para o bebê. Em nossa casuística, 100\% dos profissionais sugeriram cautela na prescrição de medicamentos. Nesse momento a gestante pode se encontrar com a pressão arterial alterada ou diminuída, exigindo mais atenção do profissional na hora de prescrever algum medicamento, que pode influenciar na formação da criança. Nesse contexto, um trabalho realizado em São Paulo em 2008 com 37 profissionais revelou que 29,70\% dos dentistas consideram importante uma consulta prévia com o profissional obstetra da paciente antes de prescrever a medicação ${ }^{17}$.

Sobre o atendimento a gestantes, todos os cirurgiões-dentistas de nosso estudo concordaram com a necessidade de cursos de capacitação para os profissionais, para melhor atendê-las. Numa pesquisa com 259 participantes realizada em 2012, 81,80\% mostraram interesse em realizar educação continuada relacionada à gestação ${ }^{9}$. Já outro estudo realizado em
CECHINEL DB

BOFF WM

CERETTA RA

SIMÕES PW

CERETTA LB

SONEGO FGF

SI STEMATIZAÇÃO

DE UM PROTOCOLO

DE ATENDIMENTO

CLÍNICO

ODONTOLÓGICO A

GESTANTES EM UM

MUNICÍPIO SUL

CATARINENSE
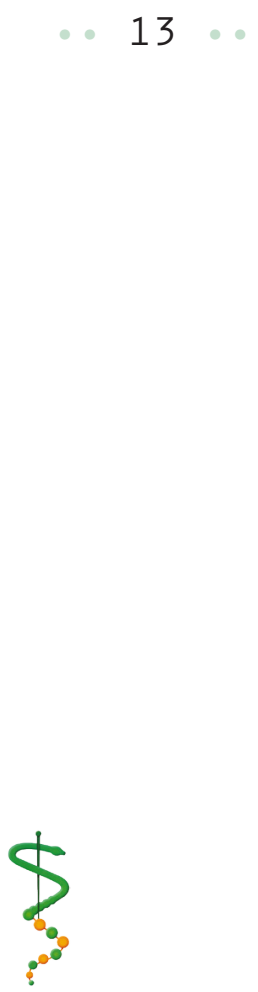

REV, ODONTOL.

UNIV, CID, SÃO

PAULO

2016; 28(1): 6-16, JAN-ABR 
CECHINEL DB

BOFF WM

CERETTA RA

SIMÕES PW :

CERETTA LB

SONNEGO FGF

SI STEMATI ZAÇÃO DE UM PROTOCOLO

DE ATENDIMENTO

CLÍNICO

ODONTOLÓGICO A GESTANTES EM UM

MUNICÍPIO SUL

CATARINENSE

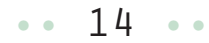

REV. ODONTOL

UNIV, CID . SÃO

PAULO

$2016 ; 28(1): 6-16$

JAN-ABR
2014 na Universidade de Ciências Médicas (localizada no Irã), com 29 alunos de obstetrícia, revelou após um curso de três meses, que houve melhora no que diz respeito aos assuntos relacionados à gestante, sendo que antes do curso eles foram questionados se havia alguma razão para dentistas não tratarem mulheres grávidas. Antes, 21,10\% concordavam que não havia nenhuma razão, após o curso 95,50\% concordavam que não havia nenhuma objeção apara atender as gestantes ${ }^{18}$.

Questionamos também se a criação de um protocolo básico para o dia a dia clínico do atendimento odontológico à gestante seria relevante? Dos entrevistados, $100 \%$ concordaram que sim, que seria relevante. Convergindo com essa questão, uma pesquisa realizada no Ceará em 2009, com a equipe de saúde da família, corrobora com nosso estudo, pois em tal estudo foi elaborado um fluxograma para atendimento odontológico na assistência ao pré-natal, além do protocolo clínico ${ }^{19}$. A secretaria Municipal de Saúde Pública de Curitiba, em 2004, também adotou o uso de um protocolo integrado de atenção à saúde bucal, objetivando, com esse instrumento de trabalho, melhores condições de saúde bucal e geral para a população Curitibana ${ }^{20}$.

A partir dos dados apresentados em nosso estudo, foi possível observar a satisfação dos participantes por meio do $2^{\circ}$ questionário, pois $55,50 \%$ concordaram que o protocolo deixou-os mais seguros para realizar os procedimentos; ao mesmo tempo, o protocolo modificou algo na vida profissional de 33,30\% dos entrevistados. Em contrapartida, quando pergun- tamos se o protocolo superou suas expectativas, aproximadamente $55,00 \%$ dos profissionais relataram que não, mas, na questão seguinte, quando questionados se o protocolo lhes proporcionou satisfação profissional, 55,30\% responderam afirmativamente. Tal divergência pode ser explicada pela própria insegurança ou medo dos profissionais em atender pacientes que estejam no período gestacional.

\section{CONCLUSÃO}

O conhecimento dos cirurgiões-dentistas sobre as alterações que ocorrem na gravidez é importante, para poder prever possíveis problemas, possibilitando, assim, um tratamento odontológico mais seguro e eficaz. Por mais que os mitos e inverdades ainda se tornem presentes ao se realizar o atendimento odontológico a gestantes, nosso trabalho mostrou que o uso de um protocolo clínico no dia a dia proporcionou mais segurança nos atendimentos, como também satisfação profissional aos cirurgiões-dentistas.

No entanto, devido ao limitado tamanho da amostra, sugere-se que futuros estudos acerca desse tema sejam realizados com mais profissionais, até mesmo correlacionando outras cidades, e outros profissionais da área da saúde.

Sugerimos, ainda, que este protocolo de atendimento básico para gestantes seja aplicado com mais profissionais que atuam em outras regiões, fazendo com que as gestantes se sintam seguras ao realizar os procedimentos odontológicos e que o profissional possa, assim, inserir novos hábitos que venham proporcionar mais qualidade de vida para a mãe e seu futuro bebê. 
1. Echeveria S, Politano G. Tratamento odontológicos para gestantes. São Paulo: Santos; 2011.

2. Santos Neto ET, Oliveira AE, Zandonade E, Leal M C. [Access to dental care during prenatal assistance]. Cien Saude Colet 2012 Nov;17(11):3057-68.

3. Cabral MCB, Santana Santos T, Moreira TP. Percepção das gestantes do Programa de Saúde da Família em relação à saúde bucal no município de Ribeirópolis, Sergipe, Brasil. Rev port saúde pública 2013 31(2):160-7.

4. Oliveira EC, Lopes JMO, Santos PCF, Magalhães SR. Atendimento Odentológico a gestantes: a importância do conhecimento da saúde bucal. RIUVRV 2014 4(1):11-23.

5. Oliveira JFM, Gonçalves PE. Verdades e mitos sobre o atendimento odontológico da paciente gestante. Rev Port Estomatol Med Dent Cir Maxilofa 2009 50(3):165-71.

6. Aljulayfi I, Alrusayni A, Alqahtani S, Hamam MK. Awareness of dental interns in managing cases of pregnant women in Saudi Arabia. Saudi J Dent Res 2015 6(1):26-9.

7. Ebrahim ZF, Oliveira MCQ, Melo Peres MPS, Franco JB. Dental treatment during pregnancy. Sci in Health 2014 5(1):32-44.

8. Martins LO, Pinheiro RPdS, Arantes DC, Nascimento LS, Santos Júnior PB. Assistência odontológica à gestante: percepção do cirurgião-dentista. Rev Pan-Amazônica Saúde (Online) 2013 4(4):11-8.

9. Luc E, Coulibaly N, Demoersman J, Boutigny $\mathrm{H}$, Soueidan A. Enquête sur la prise en charge de la femme enceinte au cabinet dentaire. Rev Mens Suisse Odontostomatol 2012 122(1047-55.

10. Patil S, Thakur R, K M, Paul ST, Gadicherla P. Oral Health Coalition: Knowledge, Attitude, Practice Behaviours among Gynaecologists and Dental Practitioners. Journal of international oral health : JIOH 2013 Feb;5(1):8-15.
11. George A, Shamim S, Johnson M Dahlen $\mathrm{H}$, Ajwani $\mathrm{S}$, Bhole $\mathrm{S}$, et al. How do dental and prenatal care practitioners perceive dental care during pregnancy? Current evidence and implications. Birth (Berkeley, Calif) 2012 Sep;39(3):238-47.

12. Strafford KE, Shellhaas C, Hade EM. Provider and patient perceptions about dental care during pregnancy. The journal of maternal-fetal \& neonatal medicine : the official journal of the European Association of Perinatal Medicine, the Federation of Asia and Oceania Perinatal Societies, the International Society of Perinatal Obstet 2008 Jan;21(1):63-71.

13. Suri V, Rao NC, Aggarwal N. A study of obstetricians' knowledge, attitudes and practices in oral health and pregnancy. Education for health (Abingdon, England) 2014 Jan-Apr;27(1):51-4.

14. Hashim R, Akbar M. Gynecologists' knowledge and attitudes regarding oral health and periodontal disease leading to adverse pregnancy outcomes. Journal of International Society of Preventive \& Community Dentistry 2014 Dec;4(Suppl 3):S166-72.

15. Caneppele TMF, Yamamoto EC, Souza AC, Valera MC, de Araújo MAM. Conhecimento dos cirurgiões-dentistas sobre o atendimento de pacientes especiais: hipertensos, diabéticos e gestantes. Journal of Biodentistry and Biomaterials, São Paulo 2011 1):31-41.

16. Chi DL, Milgrom P, Carle AC, Huebner CE, Mancl LA. Multilevel factors associated with dentists' counseling of pregnant women about periodontal health. Special care in dentistry : official publication of the American Association of Hospital Dentists, the Academy of Dentistry for the Handicapped, and the American Society for Geriatric Dentistry 2014 Jan-Feb;34(1):2-6.
CECHINEL DB

BOFF WM

CERETTA RA

SIMÕES PW

CERETTA LB

SONEGO FGF

SISTEMATIZAÇÃO

DE UM PROTOCOLO

DE ATENDIMENTO

CLÍNICO

ODONTOLÓgICO A

GESTANTES EM UM

MUNICÍPIO SUL

CATARINENSE
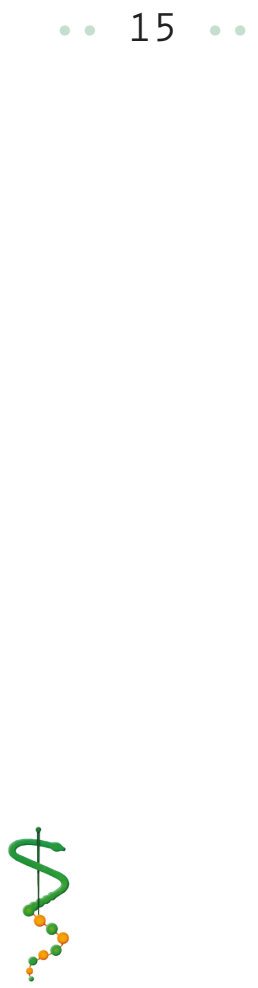

REV, ODONTOL.

UNIV, CID, SÃo

PAULO

2016; 28(1): 6-16, JAN - ABR 
CECHINEL DB BOFF WM CERETTA RA

SIMÕES PW

CERETTA LB

SONNEGO FGF

SI STEMATIZAÇÃO DE UM PROTOCOLO

DE ATENDIMENTO

CLÍNICO

ODONTOLÓGICO A GESTANTES EM UM MUNICÍPIO SUL CATARINENSE

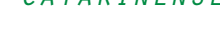

17. Zanata RL, Fernandes KB, Navarro PS. Prenatal dental care: evaluation of professional knowledge of obstetricians and dentists in the cities of Londrina/ PR and Bauru/SP, Brazil, 2004. Journal of applied oral science : revista FOB 2008 May-Jun;16(3):194-200.

18. Mohebbi SZ, Yazdani R, Sargeran K, Tartar Z, Janeshin A. Midwifery students training in oral care of pregnant patients: an interventional study. J Dent 2014 Sep;11(5):587-95.
19. Lima MCL. Implantação do atendimento odontológico à gestante na ESF do Camará em Aquiraz-CE. Especialização em Práticas Clínicas em Saúde da Família. Fortaleza, Ceará: Escola de Saúde Pública 2009.

20. Curitiba SMdSd. Protocolo integrado de Atenção à Saúde Bucal. 1 ed. Curitiba, PR: Prefeitura Municipal de Curitiba/PR; 2004.

Recebido em 02/03/2016

Aceito em 08/08/2016 